\title{
Probabilistic pattern of risks in company's Quality management system
}

\author{
Vladimir Mager ${ }^{1}$, Tatyana Leonova ${ }^{2}$, and Liudmila Chernenkaya $^{1 *}$ \\ ${ }^{1}$ Peter the Great St. Petersburg Polytechnic University, Highest School of Cyberphysics, 195251 St.Petersburg, Russia \\ ${ }^{2}$ St. Petersburg State University of Economics, Economics and Quality Department, 191023 Sadovaya str., 21, St. Petersburg, Russia
}

\begin{abstract}
One possible approach for calculation of probabilistic rate of risks in Quality management system (QMS), which is prescribed by requirements of International Standard ISO 9001:2015, is proposed in this article. Aspects of the theory of dependability and Markov techniques are used, which are applied for evaluation of probability of failures in complicated technical systems. Representation of QMS processes as a graph with controlled discrete Markov chains is suggested, which allows to evaluate a probability of customer requirements non-fulfillment as a function of an intensity of mistakes that bring to non-conformities in QMS.
\end{abstract}

\section{Introduction}

Risk-oriented approach has been announced by the new version of International standards ISO 9000 series (from 2015). Item 6.1 of the standard ISO 9001:2015 [1] prescribes to use this approach during development, implementation and continuous enhancement of the company's Quality Management System (QMS).

Risks could be caused by people (inside and outside of the company), by machines used in the production, by inappropriate management of processes, by the influence of external environment. These risks always accompany any kind of activities and depend on lot of factors. Obviously, the mathematical decision of the task for risks estimation needs attraction of methods of multidimensional statistics. But the task for optimal management of a company with consideration of risks has to be solved by decision-makers, and usually carried on the level of intuition.

The definition of the term "risk" one can find in the item 1.1 of ISO Guide 73:2009 [2]: "effect of uncertainty on objectives", where "an effect is a deviation from the expected - positive and/or negative", which means that estimation of risks could be considered as evaluation of possible scenarios by using of well-known tools from strategic management, like SWOT-analysis. Note 4 of ISO Guide 73:2009 explains that "risk is often expressed in terms of a combination of the consequences of an event (including changes in circumstances) and the associated likelihood", and the word "likelihood" (3.6.1.1) "is used to refer to the chance of something happening ... and described using general terms or mathematically (such as a probability)". So, for more accurate, scientific-based estimation of risks, the value of a risk could be reflected in terms of probability for example, in tasks of designing and maintaining of complicated technical systems, which must assure the survival of people.

Studies concerning calculation of the rate of risks in QMS have been done by many authors $[3,4,5]$, but procedures of calculations still not introduced. The aim of this report is to provide simple probabilistic procedure for estimation of organizational risks based on the analogy with conceptual approach of the theory of dependability, which is used for calculation of a level of valid level of risk in conditions of partial uncertainty.

\section{Rational}

The most important notion in the theory of dependability is a fault. Generally, a fault is explained as an event, which brings to the situation of overall or partial loss of capabilities of an object to perform its' specified functions. In organizational activities we can meet initiated faults, which depend on mistakes in decisionmaking. Let's try to apply basics of the theory of dependability for "constructing" of the simplified procedure, which could be helpful for estimation of organizational risks.

First, we'll consider "company" as a production system, consisting of various processes, interrelating with each other and aimed on performance of customer requirements. These processes should be defined by QMS and monitored in order to assure the quality of production.

Second, we'll adopt the admission that the flow of faults in a system is the simplest, and faults are random and independent events, thus a probability $P_{s}(t)$ of trouble-free operation in time of a complex system with linear structure of elements will be equal to a product of probabilities of trouble-free operation in time of system's elements [6]: 


$$
P_{c}(t)=p_{1}(t) \cdot p_{2}(t) \cdot \ldots \cdot p_{N}(t)=\prod_{i=1}^{N} p_{i}(t),
$$

where $p_{i}-$ a probability of trouble-free operation of element $i$, and $N$ - the number of elements in a system.

Third, we'll adopt the condition of stationarity of a flow of faults, which means that $p_{i}(t)=e^{-\lambda_{i} t}$, where $\lambda_{i}$ is the intensity of faults of an element $i$.

Hence, in order to calculate a probability of a complex system with linear structure of elements, it is needed to define intensities of faults of each system's element. Evident, that number of faults during selected time interval is a statistical indicator, which should be available from records on system exploitation.

Such task becomes more difficult, if the structure of a system is not linear, i.e. elements of a system are interacting in pairs, in crosses, in feedbacks. In that case the simplest formula (1) will be incorrect, and calculation of probabilities must be done in accordance with the structure of a system.

The important condition in analysis and calculations of probabilities in complex systems is an assumption that at the initial moment, in the beginning of analysis, a system was workable. It allows attracting of Markov techniques into the calculation of dependability. These techniques are widely used in the areas dealing with random processes, which reflects randomized changes of a system's states in time (or in respect to some other argument), described by random functions.

Using of Markov processes in analysis of dependability is recommended by International Standard IEC 60300-3-1:2003 [7]. In particular, the standard tells that introduction of system's behavior through Markov model requires the defining of all possible states of a system, preferably depicted in a scheme (diagram) of states and transitions. Besides, the intensities of transitions from one state to other must be determined, which makes possible to calculate the probability of staying of a system in a present set of states. Moreover, International Standard IEC 61165:1995 [8] specifies the main advantage of application of Markov techniques: "possibility to obtain flexible probabilistic model for analysis of system's behavior..."

The above mentioned assumption about the workable state of a system at the initial moment of analysis could be interpreted as a random process without aftereffects, which is characterized by transitional probabilities depending only of a previous state of a system, regardless to the way of coming (or brining) of a system to those previous state: $P_{i / i+1}=f(S)$. Selection of anchors in behavior of a system, that is determined, for instance, by periods of managerial monitoring or corrective actions, brings to splitting of the random process into discrete states, and this random process is considered as random sequence. If a random sequence has Markov properties, it is named as Markov chain, and if states of a system are divided in time, we come to discrete Markov chain, in which a system can move from one state to the other by leap [9].

Markov chain is characterized by:

(a) matrix of transitional probabilities:

$$
\left\|P_{i j}\right\|=\left(\begin{array}{llll}
P_{11} & P_{12} & \ldots & P_{1 n} \\
P_{21} & P_{22} & \ldots & P_{2 n} \\
\ldots & \ldots & \ldots & \ldots \\
P_{n 1} & P_{n 2} & \ldots & P_{n n}
\end{array}\right),
$$

(b) vector of initial probabilities, which describes the initial state of a system:

$$
P^{(0)}{ }_{\langle n\rangle}=\left\langle P_{01}, P_{02}, \ldots, P_{0 n}\right\rangle
$$

If we can effect on values of transitional probabilities, we have the controlled Markov process. From this standpoint, the combination of measures aimed on enhancement of quality in frames of QMS could be described as controlled Markov process and introduces by discrete Markov chain.

Some notes from the theory: Markov chains, depending of possibilities to move from one of states to another, could be absorbing or ergodic. The absorbing chain contains an absorb state, where a process, while reaching it, never leaves it, and thus, stops. Ergodic, or returning Markov chain, represents many of states related by matrixes of transitional probabilities, i.e. a process can move from any state to any of others through final number of steps, and do not include absorbing states. Thus, a process, described by ergodic chain, being started in some state, will never stop. From that standpoint, exactly this type of Markov processes is applicable to QMS, taking in consideration the concept of continuous improvement according with Total Quality Management and the PDCA (Deming) cycle.

Each step of ergodic process, i.e. the transition of a system from one specified state to any other state, could be characterized by transitional probabilities. Transitional probability lies on the interval $0 \leq P_{i j} \leq 1$, it is equal to zero if the transaction from $S_{i}$ to the other state $S_{j}$ is impossible by one step, and it is equals one if transaction from $S_{i}$ is impossible to any states, which means that a system "delays" in $S_{i}$, so, $P_{i i}=1$.

Mathematic model of ergodic Markov chain of a random process could be built if there are determined:

(a) limited set of alternatives $K_{i}$, where $i \in S$ - number of the state of a system;

(b) matrixes of transitions $\Pi_{[s]}^{(k)}$, relevant to changes in a system after impact $k$, named as partial control;

(c) matrix of result $R_{[S]}{ }^{(k)}$, relevant to changes in a system after impact $k$.

Partial controls in case of QMS are concrete managerial decisions, which should bring to the overall result (improvement of QMS) after limited steps of control due to the chosen management strategy.

Let's use the advice from IEC 60300-3-1:2003 [7] and try to draw a graph of states and transitions. The simplest graph introducing a system with two states $S_{1}$ and $S_{2}$ looks like following:

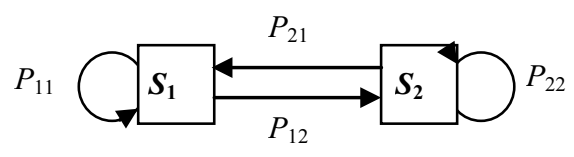


For this graph, matrix of transitional probabilities includes two lines:

$$
\left\|P_{i j}\right\|=\left(\begin{array}{ll}
P_{11} & P_{12} \\
P_{21} & P_{22}
\end{array}\right) .
$$

Here, the condition $\sum_{j=1}^{n} P_{i j}=1$ is compulsory.

Using this sample, theoretically the graph of any kind of a system could be drawn. For example, let's imagine that the sequence of processes of a production system introduced by a graph Fig. 1:

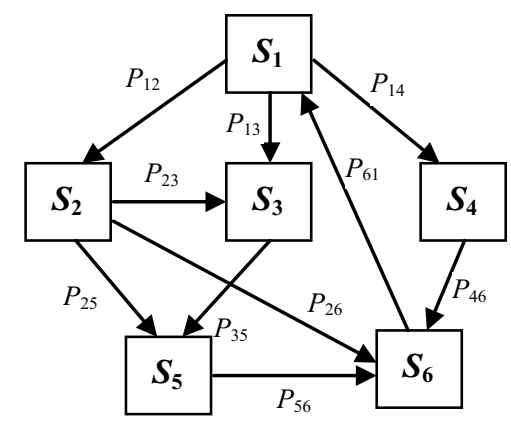

Fig. 1. Graph of states and transitional probabilities of production system:

$S_{1}$ - design, $S_{2}$ - purchases, $S_{3}-$ development of technological documentation, $S_{4}$ - planning, $S_{5}$ - technological preparation of production, $S_{6}$ - production process.

Generally, transitional probabilities for the graph Fig. 1 are determined as:

$$
\begin{aligned}
& P_{11}=1-\left(P_{12}+P_{13}+P_{14}\right) ; \\
& P_{22}=1-\left(P_{23}+P_{25}+P_{26}\right) ; \\
& P_{33}=1-P_{35} \\
& P_{44}=1-P_{46} ; \\
& P_{55}=1-P_{56} \\
& P_{66}=1-P_{61} .
\end{aligned}
$$

These equations specify a matrix of transitional probabilities, including probabilities of delays of a system in states $S_{\mathrm{j}}(\mathrm{j}=1, \ldots, \mathrm{n})$. If we define the initial state of the system as $P_{1}(0), P_{2}(0), \ldots P_{n}(0)$, it's easy to find probabilities of states $P_{1}(k), P_{2}(k), \ldots P_{n}(k)$ after any of steps $k$ :

$$
P_{i}(k)=\sum_{j=1}^{n} P_{j}(k-1) P_{i j}, \quad i=1,2, \ldots, n .
$$

This logic could be useful for selecting of optimal strategy, which has to assure maximal effectiveness of QMS. For this, matrix of transitional probabilities may be added by matrix of the same dimension with elements reflecting assumed rates of incomes or expenses, which are linked to transitions of a system between states. As for expenses, they could be related to costs for corrective actions, or implementation of managerial decisions, or loosed benefit due to the delay with transition of a system to the desired state.

But it is evident that in real production all mentioned in Fig. 1 processes are interacting in strict sequence. Initial state is $S_{1}$, and final $-S_{6}$, resulted in performing of customer order in accordance with his requirements, i.e. the completion of the production of required volumes of goods/services in time. For moving from the state $S_{1}$ to the state $S_{6}$ four steps are needed:

- step 1: transitions from the state $S_{1}$ to states $S_{2}, S_{3}, S_{4}$;

- step 2: transitions from the state $S_{2}$ to states $S_{3}, S_{5}$, and from the state $S_{4}$ to $S_{6}$;

- step 3: transitions from the state $S_{3}$ to $S_{5}$ and from $S_{2}$ to $S_{6}$;

- step 4: transitions from the state $S_{5}$ to $S_{6}$ and from $S_{6}$ to $S_{1}$.

Initial probability $P_{11}(0)=1$. Let's define the transitional probabilities in steps. Taking into consideration only states, from which the direct transition to the following state is possible, we find:

after step 1:

$$
P_{22}(1)=P_{11}(0) \cdot P_{12} \text {; }
$$$$
P_{33}(1)=P_{11}(0) \cdot P_{13} \text {; }
$$$$
P_{44}(1)=P_{11}(0) \cdot P_{14} \text {; }
$$$$
P_{33}(2)=P_{33}(1)+P_{22}(1) \cdot P_{23} \text {; }
$$

after step 2:

$$
P_{55}(2)=P_{22}(1) \cdot P_{25} \text {; }
$$$$
P_{66}(2)=P_{44}(1) \cdot P_{46} \text {; }
$$

after step 3:

$$
P_{55}(3)=P_{55}(2)+P_{33}(2) \cdot P_{35} \text {; }
$$

$$
P_{66}(3)=P_{66}(2)+P_{22}(1) \cdot P_{26} \text {; }
$$

after step 4:

$$
\begin{aligned}
& P_{66}(4)=P_{66}(3)+P_{55}(3) \cdot P_{56} \\
& P_{11}(4)=P_{66}(3) \cdot P_{61} .
\end{aligned}
$$

As the result, we have the expression for evaluation of the probability of the successful completion of company's work on the customer order:

$$
\begin{aligned}
P_{\text {success }}= & P_{66}(4)=P_{14} \cdot P_{46}+P_{12} \cdot P_{26}+ \\
& +\left[P_{12} \cdot P_{25}+\left(P_{13}+P_{12} \cdot P_{23}\right) \cdot P_{25}\right]
\end{aligned}
$$

Here, transitional probabilities $P_{i j}$ characterize the reducing of probability of moving from the state $i$ to the state $j$ because of some faults. Now we come to most important question in our proposed analogy: how to treat the term "fault" in Quality management and QMS processes?

Processes of a company are organized for performing of generalized process of production of goods and/or services, which is applicable to any kinds of production and consist of a certain consequence of separate processes [10], and each of them could be introduced as controlled discrete Markov chain. There are tens of processes in companies, and they are interacting - this is the meaning of process approach according with standard ISO 9000 [11]. Technical term "fault" in process-oriented company can be interpreted as the term "nonconformity", defined in the vocabulary of the standard ISO 9001:2015 [1] as "failure to comply with requirement". Prof. Edwards Deming was the first in $1980^{\text {th }}$, who used this term, and he associated nonconformity with delays, downtimes, mistakes, defects - all causes, which do not allow a company to carry out a production process properly. Thus, nonconformity in QMS is the analog of the fault in technical systems. It means that concepts of the theory of dependability could be applied for evaluation of risks in 
QMS, based on probabilistic models. Even though the term "probability" includes some proximity, anyway, it is more reliable to choose a strategy on digital indicators than on some low reasoned hypotheses.

Let's illustrate the simplest method of calculation of risks in QMS, taking into account all assumptions, which we adopted to the considered class of Markov chains. For this, we must define transitional probabilities in (3) and list possible mistakes belonging to arrows of the graph. In general, the probability of a mistake, in case of stationarity, could be calculated as:

$$
P_{\text {Mistake.z }}=e^{-\lambda_{\text {Mistake.z }} t_{\text {step } k}} \text {, }
$$

where $\lambda_{\text {Mistake.z }}-$ intensity of mistake $z$;

$z$ - number of mistake from the list;

$k$ - number of the step (in our example $1 \leq k \leq 4$ );

$t_{\text {step } k}$ - normative time for transition on step $k$.

List of mistakes should be completed by managers, who are in charge of particular processes. Intensities $\lambda_{\text {Mistake. }}$ have to be calculated on the basis of the data from QMS monitoring.

Most part of mistakes in QMS are related to human factor. Let's try to list some of them:

1) mistakes in design, including development of operational manuals;

2) mistakes in planning and organization of production;

3) mistakes in marketing and logistics, which causes inappropriate choose of suppliers;

4) mistakes in production, caused by divergences from requirements of design/technological documentation;

5) breach of requirements of operational manuals on equipment by personnel;

6) inappropriate organization of working places;

7) mistakes in maintenance, which causes inappropriate adjustment and calibration of technological equipment;

8) mistakes in technical inspection, caused by using of incorrect instruments or misconduct of inspection and documenting procedures;

9) misconduct of conditions for storing and shipment of final production;

10) managerial mistakes, including poor motivation of employees.

Obviously, that the whole list of mistakes is much longer, but we'll use this one to finalize our pattern:

$$
\begin{aligned}
& P_{12}=P_{13}=P_{14}=1-e^{-\lambda_{\text {Mistake. } 1} t_{\text {step } 1}} \text {; } \\
& P_{23}=P_{25}=1-e^{-\lambda_{\text {Mistake.3 }} t_{\text {step } 2}} \text {; } \\
& P_{26}=1-e^{-\lambda_{\text {Mistake. } 9} t_{\text {step } 2}} \text {; } \\
& P_{35}=1-e^{-\lambda_{\text {Mistak.7 }} t_{\text {step } 3}} ; \\
& P_{46}=1-e^{-\lambda_{\text {Mistake.2 }} t_{\text {step } 3}} \text {; } \\
& P_{56}=1-e^{-\lambda_{\text {Mistake.4 }} t_{\text {step } 3}} \text {; } \\
& P_{61}=1-e^{-\lambda_{\text {Mistake.8 }} t_{\text {step } 3}}-e^{-\lambda_{\text {Mistake.10 }} t_{\text {step } 3} 3} \text {. }
\end{aligned}
$$

This pattern of probabilities, along with (3), allows to calculate the probability of risk of non-fulfillment (or poor quality) of the work, in percentage:

$$
P_{\text {Risk }}=\left(1-P_{\text {success }}\right) \cdot 100 \% \text {. }
$$

\section{Conclusion}

The proposed approach allows to obtain a quantative rate of risk in QMS. If we are able to evaluate negative consequences of nonconformities (like failures, shutdowns, breaches of technological discipline, delays in deliveries from suppliers, etc.), founded value $P_{\text {Risk }}$ could be easily transferred into economic categories.

\section{References}

1. ISO 9001:2015. Quality management systems. Requirements

2. ISO Guide 73:2009. Risk management. Vocabulary

3. V.V. Okrepilov, Quality management (Science, St. Petersburg, 2007).

4. T.I. Leonova, Statistical methods in management of processes: handbook (Printing house of State Economic University, St. Petersburg, 2014).

5. A.V. Chernenkii, International Research Journal, 8(50), 1, 92-96 (2016)

6. V.A. Ostreikovskii, Theory of dependability: handbook for HEI (High school, Moscow, 2003)

7. IEC 60300-3-1:2003. Dependability management Part 3-1: Application guide - Analysis techniques for dependability - Guide on methodology

8. IEC 61165:1995. Application of Markov techniques

9. E.V. Berezhnaia. Mathematic methods in modeling of economic systems: handbook (Finances and Statistica, Moscow, 2006)

10. V.E. Mager, Quality management: handbook (INFRA-M, Moscow, 2015)

11. ISO 9000:2015. Quality management systems. Fundamentals and vocabulary 